\title{
During Hospital or Clinic Visits after Abnormal Screening or Diagnosis
}

National Cancer Institute

\section{Source}

National Cancer Institute. During Hospital or Clinic Visits after Abnormal Screening or

Diagnosis. NCl Thesaurus. Code C148492.

The time period during a hospital or clinic visit and after when an individual received an abnormal screening or diagnosis. 